\title{
Eculizumab Maintenance and the Prevention of Atypical Hemolytic Uremic Syndrome Relapse During Pregnancy: A Case Report
}

\author{
Mae-Lan Winchester ${ }^{\mathrm{a}, \mathrm{c}}$, Ryan Platzbecker ${ }^{\mathrm{b}}$, Megan McMahon ${ }^{\mathrm{a}}$, Marc Parrish ${ }^{\mathrm{a}}$
}

\begin{abstract}
Atypical hemolytic uremic syndrome is a thrombotic microangiopathy that can cause life-threatening anemia, thrombocytopenia, and acute renal failure. When triggered during pregnancy or the acute postpartum period, the disease is referred to as pregnancy-associated atypical hemolytic uremic syndrome (P-aHUS). Women who suffer $\mathrm{P}$-aHUS may later want to consider future pregnancy. These patients are at high, though not well-estimated, risk of relapse. Eculizumab, a monoclonal antibody against complement 5 (C5), has been highly successful in treating acute attacks of P-aHUS, but little is known about the effectiveness of eculizumab maintenance therapy throughout pregnancy. In this case report, we present a woman whose first pregnancy was complicated by severe P-aHUS. In her next pregnancy, she was maintained on eculizumab. She delivered a healthy infant at term and had no recurrences of P-aHUS during the pregnancy or in the postpartum period.
\end{abstract}

Keywords: Pregnancy; aHUS; Eculizumab

\section{Introduction}

Atypical hemolytic uremic syndrome (aHUS) is a rare disease that has a high degree of morbidity and mortality if not treated promptly. Pregnancy can occasionally serve as a trigger, and pregnancy-associated aHUS (P-aHUS) has been estimated to complicate 1 in 25,000 pregnancies [1]. Patients with a history of P-aHUS are at increased risk of relapse during subsequent pregnancy. Eculizumab is a terminal complement inhibitor that received the approval from the Food and Drug Administration (FDA) for treatment of aHUS in 2011. It has been successfully

Manuscript submitted November 29, 2019, accepted December 9, 2019

aDepartment of Obstetrics and Gynecology, Kansas University Medical Center, Kansas City, KS 64113, USA

b Department of Internal Medicine, KC Hospitalists, 4831 W 136th St., Unit A Leawood, KS 66224, USA

${ }^{\mathrm{c} C o r r e s p o n d i n g ~ A u t h o r: ~ M a e-L a n ~ W i n c h e s t e r, ~ D e p a r t m e n t ~ o f ~ O b s t e t r i c s ~ a n d ~}$ Gynecology, Kansas University Medical Center, Kansas City, KS 64113, USA. Email: mwinchester@kumc.edu

doi: https://doi.org/10.14740/jmc3397 used in pregnancy during acute aHUS and more extensively in paroxysmal nocturnal hemoglobinuria, but little is known about the safety and efficacy of eculizumab maintenance during pregnancy.

\section{Case Report}

A 37-year-old Caucasian female presented to our maternal fetal medicine clinic with her second pregnancy at 7 weeks of gestation. Her first pregnancy had been complicated by P-aHUS. That pregnancy had been rather uneventful until 40 weeks gestation, when she was induced at an outside hospital for suspected preeclampsia, based on elevated blood pressures and an elevated protein/creatinine ratio. She had an uncomplicated vaginal delivery and was discharged home on postpartum day 2. She re-presented on postpartum day 4 complaining of headache and malaise. She was noted to have severely elevated blood pressures, anemia (hemoglobin: $5 \mathrm{~g} / \mathrm{dL}$ ), thrombocytopenia (platelets: $32 \times 10^{3} / \mu \mathrm{L}$ ), and acute kidney injury (creatinine: 7.2 $\mathrm{mg} / \mathrm{dL}$ ) later requiring dialysis. She quickly developed acute hypoxic respiratory failure requiring intubation. ADAMTS-13 (a disintegrin and metalloproteinase with thrombospondin type 1 motif, member 13) activity was obtained and ultimately returned as normal. With multidisciplinary team involvement, she was diagnosed with P-aHUS, and started on plasmapheresis and eculizumab. She experienced marked improvement in both clinical and laboratory parameters.

Since her initial diagnosis, the patient was maintained on eculizumab infusions ( $900 \mathrm{mg}$ ) every 2 weeks and experienced full recovery of renal function (creatinine: $0.6 \mathrm{mg} / \mathrm{dL}$, protein/ creatinine ratio: 0.2). Her blood pressures, however, never quite normalized and she was kept on carvedilol $6.25 \mathrm{mg}$ twice daily. She declined any genetic workup.

Aside from P-aHUS and hypertension, the patient had no other significant past medical or surgical history. She was informed of the risks of P-aHUS relapse and pregnancy complications. Given her risk of relapse during this subsequent pregnancy, she continued to receive eculizumab infusions every 2 weeks throughout the pregnancy. Complement 5 (C5) and free eculizumab levels were not available at our institution. The patient was kept on carvedilol and did not require dose increase during pregnancy. Assessment of renal function and complete blood count were obtained every 2 weeks and remained normal. Aside from gestational diabetes controlled with oral medica- 
tions, the remainder of her pregnancy was uncomplicated. Fetal size was appropriate for gestational age on routine growth ultrasounds. The patient underwent induction of labor at 39 weeks gestation and had a spontaneous vaginal delivery. She received a supplemental dose of eculizumab within $24 \mathrm{~h}$ of delivery (despite receiving her usual dose 7 days prior) as recommended by her hematologist. She then resumed infusions every 2 weeks. Pediatricians were alerted as to the mother's use of eculizumab. The healthy male infant was monitored in the full-term nursery and no evidence of infection was found during observation.

\section{Discussion}

Though rare, P-aHUS can threaten the life of both mother and fetus. Most cases occur during the woman's first pregnancy [2]. Some of these women may later desire another pregnancy. The risk of relapse during a subsequent pregnancy is thought to be high, but is not well studied. And while brief eculizumab treatment has been greatly effective at treating active disease during pregnancy, less is known about eculizumab maintenance during pregnancy [3].

Only seven pregnancies in patients previously diagnosed with $\mathrm{P}-\mathrm{aHUS}$ and maintained on eculizumab are reported in the literature [4-6]. Of these seven patients, only one patient experienced an uncomplicated pregnancy and delivered at term. The remaining six pregnancies were all delivered before 34 weeks gestation. Two developed P-aHUS relapse, one suffered an intrauterine fetal demise at 24 weeks, and the remaining three developed preeclampsia/HELLP (hemolysis, elevated liver enzymes, and low platelets) syndrome. Only one patient received a supplemental dose of eculizumab within $24 \mathrm{~h}$ of delivery, but this was after development of HELLP syndrome with concerns for full P-aHUS relapse. Our patient received a supplemental eculizumab dose as a form of prophylaxis.

In limited human data, eculizumab has not been found to be associated with congenital anomalies, increased risk of stillbirth, neonatal death, or serious neonatal infectious morbidity. Indeed, complement activity has been measured in the serum of babies born to mothers treated with eculizumab and those that were not treated [7]. Similar complement levels were found, which suggests that eculizumab does not affect the neonatal complement syndrome. The infant born to the presented patient did not demonstrate any concerns for potential infection, and complement levels were not checked after birth. More data are needed to confirm the safety of eculizumab maintenance during pregnancy for both the mother and fetus. However, given the life-threatening nature of P-aHUS to both mother and fetus, eculizumab maintenance therapy during pregnancy may confer more benefit than risk to the dyad.

\section{Acknowledgments}

None.

\section{Financial Disclosure}

None to declare.

\section{Conflict of Interest}

None to declare.

\section{Informed Consent}

Obtained.

\section{Author Contributions}

MW wrote the manuscript. RP, MM, and MP edited and provided expertise. All authors have read and approved the manuscript.

\section{References}

1. Huerta A, Arjona E, Portoles J, Lopez-Sanchez P, Rabasco C, Espinosa M, Cavero T, et al. A retrospective study of pregnancy-associated atypical hemolytic uremic syndrome. Kidney Int. 2018;93(2):450-459.

2. Bruel A, Kavanagh D, Noris M, Delmas Y, Wong EKS, Bresin E, Provot F, et al. Hemolytic uremic syndrome in pregnancy and postpartum. Clin J Am Soc Nephrol. 2017;12(8):1237-1247.

3. Sarno L, Tufano A, Maruotti GM, Martinelli P, Balletta MM, Russo D. Eculizumab in pregnancy: a narrative overview. J Nephrol. 2019;32(1):17-25.

4. Fontana F, Alfano G, Bardhushi E, Ligabue G, Giovanella S, Neri I, Cappelli G. Relapse of atypical hemolytic uremic syndrome during pregnancy in a patient on eculizumab maintenance treatment: a case report. Am J Case Rep. 2019;20:1460-1465.

5. Servais A, Devillard N, Fremeaux-Bacchi V, Hummel A, Salomon L, Contin-Bordes C, Gomer H, et al. Atypical haemolytic uraemic syndrome and pregnancy: outcome with ongoing eculizumab. Nephrol Dial Transplant. 2016;31(12):2122-2130.

6. Bateman S, Ladhani M, Jesudason S. Successful subsequent pregnancy in a woman receiving eculizumab for pregnancy-associated atypical haemolytic uraemic syndrome. Case Rep Nephrol. 2019;2019:2738723.

7. Hallstensen RF, Bergseth G, Foss S, Jaeger S, GeddeDahl T, Holt J, Christiansen D, et al. Eculizumab treatment during pregnancy does not affect the complement system activity of the newborn. Immunobiology. 2015;220(4):452-459. 